\title{
Wenn der Patient nicht will Was tun bei Transportverweigerung?
}

Der Patient müsste eigentlich ins Krankenhaus, z. B. weil die präklinischen Möglichkeiten nicht ausreichen, um eine ernsthafte Verletzung zu behandeln oder auszuschließen. Aber er lehnt Versorgung und Transport ab. Was dann?

Guido C. Bischof

\section{Dürfen Patienten eine Versorgung ableh-}

nen? Zumindest wenn der begründete Verdacht besteht, dass eine ernsthafte Verletzung oder Erkrankung vorliegt, erscheint es unklug, sich nicht behandeln oder in ein Krankenhaus transportieren zu lassen. Dennoch:

- Vor jeder medizinischen Maßnahme ist eine Einwilligung des Patienten einzu- holen (§ 630d Abs. 1 Bürgerliches Gesetzbuch (BGB)).

- Verweigert der Patient diese Einwilligung, ist dies grundsätzlich verbindlich - und medizinische Maßnahmen haben zu unterbleiben.

Dabei geht die Rechtsprechung grundsätzlich davon aus, dass eine Einwilligungsfähigkeit beim Erwachsenen die Regel ist [1].
Entsprechend kann der Patient medizinisch indizierte Maßnahmen ablehnen, auch wenn dies nicht sinnvoll erscheint.

Entscheidungsfähigkeit ist relevant Der Patient muss zunächst grundsätzlich und in der konkreten Situation in der Lage sein, Entscheidungen über seinen Gesundheitszustand zu treffen.

Einwilligungsfähig bzw. entscheidungsfähig ist, wer Bedeutung und Tragweite - auch die Risiken - der Maßnahme bzw. der Ablehnung der Versorgung erfassen und seinen Willen hiernach zu bestimmen vermag [2]. 
An der grundsätzlichen Einwilligungsfähigkeit bzw. Entscheidungsfähigkeit können sich verletzungs-, erkrankungs- oder situationsbedingt Zweifel ergeben. Um solche Zweifel, auch vorsorglich, auszuräumen, empfiehlt es sich, hierzu Feststellungen zu treffen und diese zu dokumentieren.

\section{Wann ist man einwilligungsfähig? Eine} einfache Dokumentation „Patient ist zurechnungsfähig“ o. ä. ist im Nachhinein wenig nachvollziehbar und kaum belastbar. Vielmehr sollte die Dokumentation anhand nachvollziehbarer Kriterien erfolgen.

- Prüfen Sie, ob der Patient zu allen 4 Qualitäten (persönlich, zeitlich, örtlich, situativ) orientiert ist.

- Fehlen dann noch Befunde, die auf eine akute neurologische oder psychiatrische Erkrankung oder eine Intoxikation schließen lassen, wird i.d.R. eine Entscheidungsfähigkeit gegeben sein.

Bei einem bewusstlosen Patienten, der aktiv keine Entscheidung mitteilen kann, ist grundsätzlich davon auszugehen, dass der Patient versorgt werden möchte.

Aufklärung tut Not Um sicherzustellen, dass der Patient eine zutreffende Vorstellung von seiner gesundheitlichen Situation und den möglichen Folgen einer Verweigerung der Versorgung hat, sollte er entsprechend informiert werden. Dabei ist dem Patienten zu erklären,

- welche Verletzung oder Erkrankung bei ihm möglicherweise vorliegt und

- was im ungünstigsten Fall die Folgen seiner Ablehnung sind.

Zurückhaltung ist hier fehl am Platz. Sprechen Sie einfach und deutlich und verzichten Sie auf medizinische Fachausdrücke.

- So sollte etwa ein Patient mit einer Kopfplatzwunde auch darüber informiert werden, dass ohne weitere Untersuchung eine intrakranielle Blutung (für den Patienten „Hirnblutung“) nicht sicher auszuschließen ist und letztlich Bewusstlosigkeit, dauernde Schäden oder der Tod drohen können.

Der Patient würde ansonsten seine Kopfverletzung möglicherweise nur für einen „kleinen Kratzer“ halten, um „den die Sanitäter soviel Aufriss machen“.

Wer darf bzw. muss aufklären? Auch heute herrscht teilweise noch die Auffassung, die Aufklärung vor einem medizinischen Eingriff könne nur durch einen Arzt erfolgen [3]. Dies geht zum einen auf das ärztliche Berufsrecht [4], zum anderen auf eine über 40 Jahre alte Entscheidung des Bundesgerichtshofs (BGH) [5] zurück. Das ärztliche Berufsrecht ist für Rettungsfachpersonal nicht bindend, bei der genannten Entscheidung spielten noch andere Faktoren eine Rolle. Dennoch wird die Meinung vertreten, die Aufklärung müsse auch durch einen Arzt erfolgen, wenn der Patient eine Versorgung bzw. einen Transport ablehnt.

Aufklärung durch Notarzt: nicht immer praktikabel Dies hätte zur Folge, dass zu jedem verweigernden Patienten ein Notarzt oder anderer Arzt nachgefordert werden müsste, damit dieser den Patienten über die Folgen seiner Verweigerung aufklärt.

- Schon praktisch scheint eine derartige Bindung der Ressource Notarzt bei oft knapper Verfügbarkeit sehr fraglich.

- Auch sehen diverse Notarztindikationskataloge, z.B. der Bundesärztekammer [6], eine solche Situation nicht als Notarztindikation an.

\section{Argumente für die Aufklärung durch den} Rettungsdienst Das BGB sieht im Patientenrechtegesetz seit 2013 zudem vor, dass

- die Aufklärung vor einem medizinischen Eingriff durch eine Person erfolgen muss, die über die zur Durchführung der Maßnahme notwendige Ausbildung verfügt.

- Dem Rettungspersonal mutet man zu, viele Verletzungen und Erkrankungen präklinisch alleine zu versorgen. Dazu ist es durch die entsprechende Ausbildung regelhaft tatsächlich befähigt.

Hierfür ist eine entsprechende Kommunikation nebst Aufklärung und Einwilligung des Patienten nötig, auch wenn man dies in Ausbildung und Praxis oft nicht so bezeichnet. Wieso ein Notarzt erforderlich sein soll, nur weil der Patient sich nicht versorgen lassen möchte, ist nicht schlüssig.

- Wenn das Rettungsfachpersonal in der Lage ist, den Patienten alleine zu versorgen, kann es ihn auch über die Folgen einer Verweigerung aufklären.

Rechtlich ist zur Aufklärung im Rettungsdienst nicht immer ein Arzt erforderlich. Die Aufklärung des verweigernden Patienten kann auch durch das Rettungspersonal erfolgen, sofern es zur Durchführung der eigentlich notwendigen Maßnahme ausgebildet ist.

Wann doch ein Arzt aufklären muss Etwaige entgegenstehende lokale Regelungen, die in einem solchen Fall das Hinzuziehen eines Notarztes vorsehen, sind natürlich zu beachten. Auch wenn sich am Einsatzort herausstellt, dass der Patient an einer Verletzung oder Erkrankung leidet, die eine Notarztindikation darstellt, sollte der Notarzt hinzugezogen werden. Dies gilt auch, wenn der Patient die Versorgung voraussichtlich ablehnen wird.

\section{Ablehnung und Dokumentation}

Konsequenzen der Ablehnung Ein Patient, der sowohl entscheidungsfähig als auch hinreichend aufgeklärt ist, kann eine Versorgung ablehnen.

- Der Patient trägt dann aber die volle medizinische und rechtliche Verantwortung.

- Problematisch sind Situationen, in denen dem Patienten, der eine Versorgung abgelehnt hat, ein Schaden entsteht. 
Unter derartigen Umständen kann es sein, dass der Patient seine Ablehnung bedauert und die Schuld beim Rettungspersonal sucht. Noch unglücklicher sind Situationen, in denen der Patient nach dem ersten Kontakt mit dem Rettungsdienst verstirbt - und Dritte Anschuldigungen erheben.

Dadurch können sich straf- und zivilrechtlich (Schadenersatz/Schmerzensgeld) Fälle ergeben, in denen man dem Rettungspersonal vorwirft, den Patienten „einfach nicht behandelt oder transportiert zu haben“. Die Folgen eines solchen Vorwurfs können gravierend sein.

Rechtliche Konsequenzen für die Mitarbeiter Aufgrund der Garantenstellung des Rettungsfachpersonals ( $\$ 13$ Strafgesetzbuch, StGB) zählt das Unterlassen einer Versorgung so, als habe das Personal dem Patienten selbst aktiv geschadet.

- Der Tatvorwurf wird daher auf Körperverletzung lauten, im Todesfall zumindest auf eine fahrlässige Tötung.

- Parallel dazu können arbeitsrechtliche Folgen bis zur Kündigung auftreten.

Bei einer Tätigkeit im öffentlichen Dienst ist in den meisten Bundesländern ausgeschlossen, dass der Geschädigte oder seine Erben die Retter persönlich zivilrechtlich in Anspruch nehmen (zweifelhaft für BadenWürttemberg). Schadenersatzansprüche sind gegenüber dem Träger des Rettungsdienstes geltend zu machen. Allerdings:

- Der Träger des Rettungsdienstes kann bei Vorsatz oder grober Fahrlässigkeit diese zivilrechtlichen Ersatzansprüche beim Mitarbeiter geltend machen.

Was tun bei Vorwürfen? Aufgrund der möglichen Folgen des Vorwurfs, einen Patienten nicht versorgt zu haben, ist es klug,

- keine Angaben gegenüber Ermittlungsbehörden (z.B. Polizei, Staatsanwaltschaft) zu machen.
- Als Beschuldigter müssen Sie nie etwas aussagen.

- Als Zeuge muss man nur vor dem Staatsanwalt oder dem Gericht aussagen, nicht vor der Polizei - Angaben zu den eigenen Personalien ausgenommen.

- Vermeiden Sie auch einen „unverbindlichen Plausch“ mit Polizeibeamten: Dieser findet sich später sorgfältig dokumentiert in der Ermittlungsakte wieder.

- Sinnvoll kann es dagegen sein, frühzeitig einen Anwalt zu kontaktieren.

Auch aus diesen Gründen gilt: Bemühen Sie sich aktiv um eine Versorgung und ggf. den Transport des Patienten und kommunizieren Sie dies an der Einsatzstelle auch so deutlich.

Richtig dokumentieren Schadensfälle klären Gerichte später über Wochen und Monate, i.d.R. durch Hinzuziehen externer Sachverständiger. Eine vollständige Dokumentation, im Idealfall mit einer vom Patienten unterzeichneten Ablehnungserklärung, ist dann Gold wert.

- Sie sollte nicht nur auf das Erkrankungsbzw. Verletzungsbild des Patienten an sich bezogen sein, sondern auch

- Ausführungen zur Entscheidungsfähigkeit des Patienten,

- zur Aufklärung und

- zur dennoch erfolgten Ablehnung der Versorgung enthalten.

Viele Einsatzprotokolle sehen auf der Rückseite ein Feld vor, auf dem der Patient und Zeugen nochmals ausdrücklich Aufklärung und Ablehnung dokumentieren können. Zur Unterzeichnung kann man den Patienten aber nicht zwingen.

- Sofern er sich weigert, das Formular zu unterzeichnen, muss die sonstige Dokumentation, ggf. nebst der Unterschrift unbeteiligter Zeugen, ausreichen.
A Sich simpel eine Unterschrift auf dem
Ablehnungsformular geben zu lassen, ist im Schadensfall nicht ausreichend. Wichtig ist eine korrekte und umfangreiche Dokumentation.

Ablehnung durch den Rettungsdienst: besser nicht! Es mag auch Fälle geben, in denen Sie eine Versorgung oder einen Transport des Patienten nicht für erforderlich halten. Der Patient nachts um 3 Uhr, der seit mehreren Wochen unter Rückenschmerzen leidet, ist hier ein schönes Beispiel.

- Die Versuchung, dem Patienten zu empfehlen, am nächsten Morgen den Hausarzt aufzusuchen, und zur Wache zurückzukehren, ist groß.

- Praktisch sind solche Fälle aber sowohl zivil- als auch strafrechtlich äußerst haftungsanfällig: Mit den beschränkten Möglichkeiten vor Ort lassen sich ernsthafte Erkrankungen selten vollständig ausschließen.

Was, wenn der Patient ablehnt, aber nicht entscheidungsfähig erscheint? Wenn ein Patient die Versorgung ablehnt, man aber Zweifel an seiner Entscheidungsfähigkeit hat, besteht Handlungsbedarf.

- Zur besseren Beurteilung der Frage der Einsichtsfähigkeit des Patienten sollte man dann einen (Not-)Arzt hinzuziehen.

- Versuchen Sie stets, den Patienten von einer Einwilligung in die Versorgung zu überzeugen.

Bei einem organisch erkrankten Patienten, der entscheidungsunfähig ist, kommt perspektivisch die Einrichtung einer rechtlichen Betreuung ( $\S \S 1896$ ff. BGB) durch das zuständige Amtsgericht infrage. Diese Entscheidung kann man aber in einer akuten Notfallsituation nicht kurzfristig abwarten.

- Es kommt dann rechtlich auch eine sog. Geschäftsführung ohne Auftrag in Betracht [7]. 
Verhalten bei psychiatrischen Patienten Sofern der Patient primär psychiatrisch behandlungsbedürftig ist, muss man

- prüfen, ob eine Behandlung nach den Gesetzen zur Behandlung psychisch Kranker bzw. den Unterbringungsgesetzen möglich ist. Hierbei handelt es sich um Gesetze des jeweiligen Bundeslands. In der Regel wird hier das Vorliegen einer psychiatrischen Erkrankung, eine Eigen- / Fremdgefährdung und ein Eilbedarf erforderlich sein.

- In einem solchen Fall ist je nach örtlicher Regel die Ordnungsbehörde bzw. Polizei hinzuzuziehen.

Keinesfalls mit Gewalt Besonderen Wert muss auf die Eigensicherung des Rettungsfachpersonals gelegt werden.

- Versuchen Sie niemals, den Patienten gewaltsam mitzunehmen.

- Sofern ein Transport gegen den erklärten Willen des Patienten unabwendbar ist, müssen Sie die Polizei / Ordnungsbehörde rufen. Diese kann den Patienten ggf. in Gewahrsam nehmen, um ihn ins Krankenhaus zu bringen [8]. Das kommt aber nur in Betracht, wenn der Patient akut erkrankt und entscheidungsunfähig ist.

\section{Fazit}

Ein verweigernder Patient kann medizinisch und juristisch herausfordernd sein. Mit einem gewissen Problembewusstsein und umfangreicher Dokumentation lässt sich der Lage aber gelassener entgegensehen.
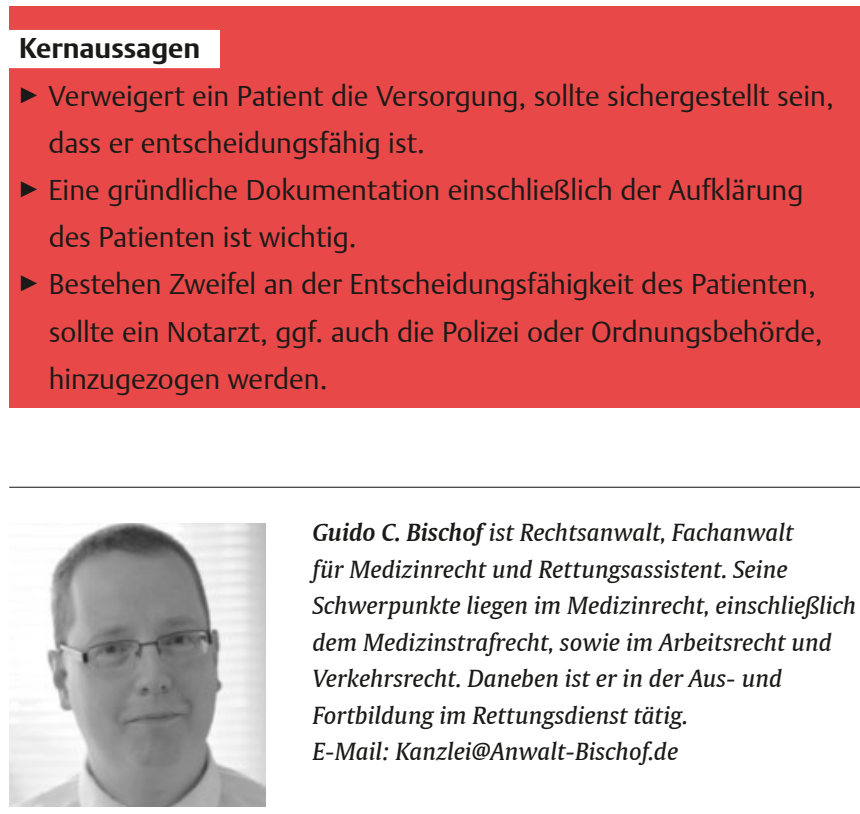

Guido C. Bischof ist Rechtsanwalt, Fachanwalt für Medizinrecht und Rettungsassistent. Seine Schwerpunkte liegen im Medizinrecht, einschließlich dem Medizinstrafrecht, sowie im Arbeitsrecht und Verkehrsrecht. Daneben ist er in der Aus- und Fortbildung im Rettungsdienst tätig. E-Mail: Kanzlei@Anwalt-Bischof.de

\section{Literatur online}

Das vollständige Literaturverzeichnis zu diesem Beitrag finden Sie online. 